\title{
A Rare Intrasplenic Varix in a Patient with Chronic Liver Disease and Portal Hypertension
}

\author{
Sivasundhar Kumarasamy ${ }^{1}$ Karumuri Srinivas Sekhar ${ }^{1}$ Malathi Vaithyanathan ${ }^{1}$ \\ Saravanakumar Sengottaiyan ${ }^{1}$ Saravanan Thangam Shanmugasundaram ${ }^{1}$
}

${ }^{1}$ Department of Radiology, Billroth Hospital, Chennai,

Tamil Nadu, India

\begin{abstract}
Address for correspondence Sivasundhar Kumarasamy, MBBS, DNB, Department of Radiology, Billroth Hospital, 43, Lakshmi Talkies Road, Shenoy Nagar, Chennai 600030, Tamil Nadu, India (e-mail: siva27sundhar@gmail.com).
\end{abstract}

\begin{abstract}
We present a rare case of spontaneous intrasplenic varix in a patient with chronic liver disease and portal hypertension. The venous collaterals and presence of portal hypertension was incidentally detected by abdominal contrast-enhanced computed tomographic imaging during evaluation of a suspected abdominal trauma following Keywords

- chronic liver disease

- portal hypertension

- intrasplenic varix a road traffic accident. There have been a few reports of splenic vein aneurysms in the extrasplenic location. To the best of our knowledge, this is the first reported case of intrasplenic varix that developed in an adult with chronic liver disease and portal hypertension which is a rare manifestation of a well-known disease.
\end{abstract}

\section{Introduction}

The portal venous circulation is a unique system which connects two different capillary beds; one in the gastrointestinal tract and splenic parenchyma, and the second in the hepatic sinusoids. Normal portal venous pressure is between 5 and $10 \mathrm{~mm} \mathrm{Hg}$, while the normal pressure gradient between the portal vein and the inferior vena cava, known as the hepatovenous pressure gradient (HVPG), is usually 1 to $5 \mathrm{~mm} \mathrm{Hg.}{ }^{1}$ Portal hypertension is defined as an abnormal pathologic increase in the portal venous pressure due to increased resistance to portal venous outflow. The portal hypertension can be classified based on the anatomical location of the resistance/obstruction, as prehepatic (involving the mesenteric, splenic, or extrahepatic portal vein), intrahepatic (liver diseases), and posthepatic (obstruction to hepatic venous drainage). The most common (>90\%) cause of portal hypertension is cirrhosis of the liver (intrahepatic causes). ${ }^{2}$ In addition, there is progressive vasodilatation in the splanchnic circulation which further aggravates the portal hypertension by augmenting portal blood flow. Recent updates suggest that the hepatic sinusoidal endothelial dysfunction also contributes to the elevation of portal venous pressure. ${ }^{1}$

Clinically significant portal hypertension is defined as an increase in HVPG to $\geq 10 \mathrm{~mm} \mathrm{Hg}$; above this threshold, the complications of portal hypertension may begin to appear. ${ }^{3}$
The increased resistance results in rerouting of blood flow away from the liver through various collateral pathways to low-pressure systemic veins. Formation of portosystemic collaterals is a complex process involving the opening, dilatation, and hypertrophy of preexisting vascular channels to decompress the portal system. ${ }^{4-8}$ Some have also postulated that a component of angiogenesis mediated by vascular endothelial growth factor is also involved in collateral formation. ${ }^{9}$ The diagnosis of pulmonary hypertension can be confirmed with a sensitivity of 70 to $80 \%$ in the presence of abdominal collateral veins. ${ }^{10,11}$

The areas of normal portosystemic anastomoses include:

1. Left gastric veins, along with esophageal veins draining into the azygos vein.

2. The superior rectal vein anastomosing with the middle and inferior rectal veins, tributaries of the internal iliac, and pudendal veins, respectively.

3. Paraumbilical and subcutaneous veins in the anterior abdominal wall.

4. Tributaries of the splenic and pancreatic veins joining the left renal vein in the retroperitoneal space.

5. Short collateral veins between splenic, colic, and lumbar veins of the posterior abdominal wall.

6. Veins in the bare area of liver communicating with diaphragmatic veins and the right internal thoracic vein. ${ }^{12}$ received

August 10, 2018

accepted after revision

August 18, 2018
DOI https://doi.org/

10.1055/s-0038-1673318.
C2018 by Indian Society of Gastrointestinal and Abdominal Radiology
License terms

(우 (1) $\ominus \circledast$ 


\section{Case Presentation}

A 47-year-old male patient presented to our emergency department after sustaining injuries in a road traffic accident the previous day. The patient had lost consciousness for 45 minutes following the trauma. He had sustained abrasions and contusions over the face, right thigh, bilateral upper limbs, anterior chest, and abdominal wall. Three months before the trauma, the patient was diagnosed elsewhere to have decompensated alcohol liver disease with grade I varices and was on treatment for alcohol dependence. There was no family history of liver disease.

On examination, the patient was found to be alert and oriented; pallor with icterus was evident without cyanosis, lymphadenopathy, or peripheral edema. A clinical examination of the abdomen revealed moderate splenomegaly. The rest of the physical examination was noncontributory. Investigations revealed anemia with thrombocytopenia, elevated bilirubin, and decreased serum albumin with relatively increased serum globulin. Tests for hepatitis B, hepatitis C, and human immunodeficiency virus were negative. Computed tomography (CT) of the brain was normal, and X-ray of the extremities revealed an undisplaced right distal radius fracture. Baseline ultrasound of the abdomen revealed a coarse echogenic liver, multiple abdominal collaterals, mild splenomegaly, and dilated intrasplenic veins without evidence of free fluid in the abdomen.

Contrast-enhanced dual source multidetector CT (Somatom Definition, Siemens, Munich, Germany) of the abdomen was performed; Precontrast and postcontrast (arterial, 35 seconds; venous, 60 seconds; delayed, 5 minutes) $1 \mathrm{~mm}$ thin sections were acquired from the domes of the diaphragm to just below the pubic symphysis following intravenous administration of low osmolar nonionic contrast, iopromide (Ultravist 370, Berlin, Germany) at the dose of $1.5 \mathrm{~mL} / \mathrm{kg}$ and rate of $3.5 \mathrm{~mL} / \mathrm{s}$.

Contrast-enhanced CT of the abdomen revealed no evidence of hemoperitoneum, pneumoperitoneum, rib fracture, or solid organ injury. The hepatic parenchyma showed heterogeneous enhancement with mild irregular surface and no

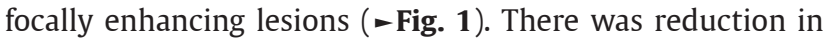

the volume of right lobe of the liver with a relatively prominent caudate lobe. Chronic thrombosis with recanalization of the right portal vein and diffusely narrow caliber main portal vein was seen along with extensive portosystemic collaterals in the periportal, peripancreatic, and left gastric regions with a lienorenal shunt (-Fig. 2). Multiple intrapancreatic collaterals were also seen with mild diffuse peripancreatic fat stranding.

The spleen was diffusely enlarged with heterogeneous, multiple, small, focal, patchy hyperdense, and hypodense foci on nonenhanced CT. An oval, circumscribed, homogeneous $6.4 \times 3.6 \times 3.2 \mathrm{~cm}$ intraparenchymal lesion paralleling the density of the splenic vein was present, suggestive of an intrasplenic venous varix ( - Figs. 3 and $\mathbf{4}$ ). The splenic artery was normal with no evidence of an arteriovenous fistula or thrombus.

\section{Management and Outcome}

Based on the MDCT appearance, the patient was diagnosed to have chronic liver disease and portal hypertension with large portosystemic collaterals and an intrasplenic varix. The patient was treated symptomatically for the trauma with one unit of blood transfusion, analgesia, bed rest, and slab immobilization of the right radius fracture. Hemoglobin levels improved and the patient recovered well. He was on regular follow-up for symptomatic hypersplenism, change in varix size, and bleeding diathesis, for 3 months.

\section{Discussion}

With the advent of MDCT, unusual pathways of portosystemic anastomoses are increasingly being recognized. Identification of such large shunts on imaging has widespread clinical implications since these shunts can cause variceal bleeding and hepatic encephalopathy. In addition, understanding their anatomy may help to avoid potential complications related to interventional radiological procedures and surgery. For patients in whom endoscopic therapy fails to control gastroesophageal variceal bleeds, interventional radiology procedures such as transjugular intrahepatic portosystemic shunt, balloon-occluded
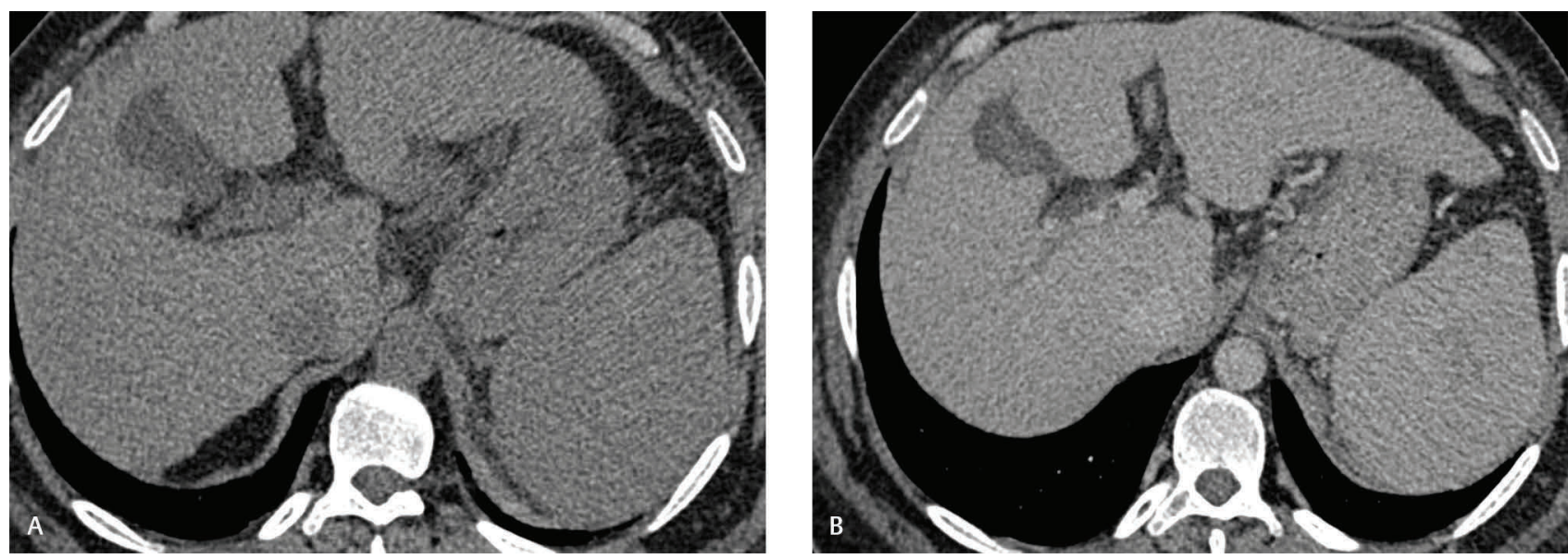

Fig. 1 Axial (A) nonenhanced computed tomography (NECT) and (B) venous phase showing heterogeneous parenchymal density of the liver. 

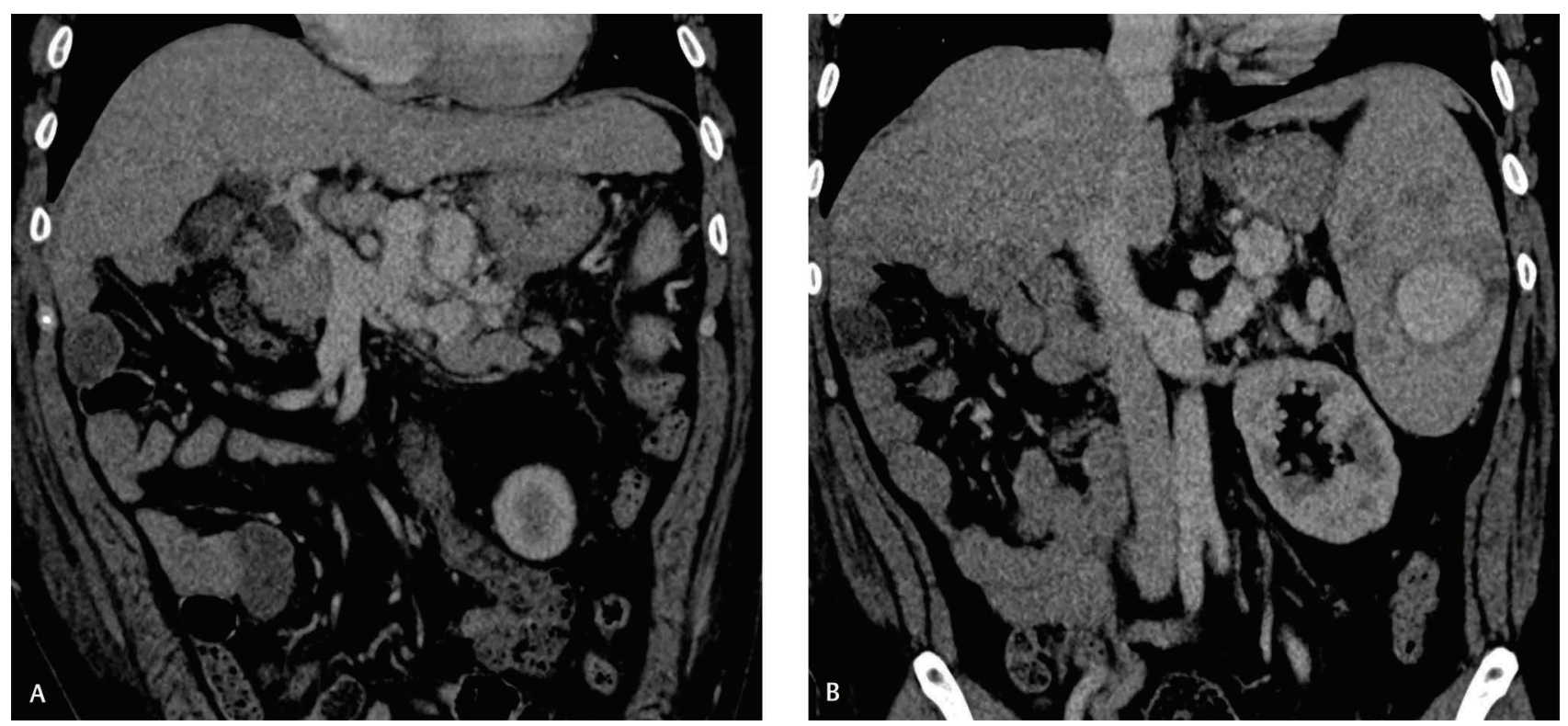

Fig. 2 (A, B) Coronal computed tomography (CT) images showing portosystemic collaterals.
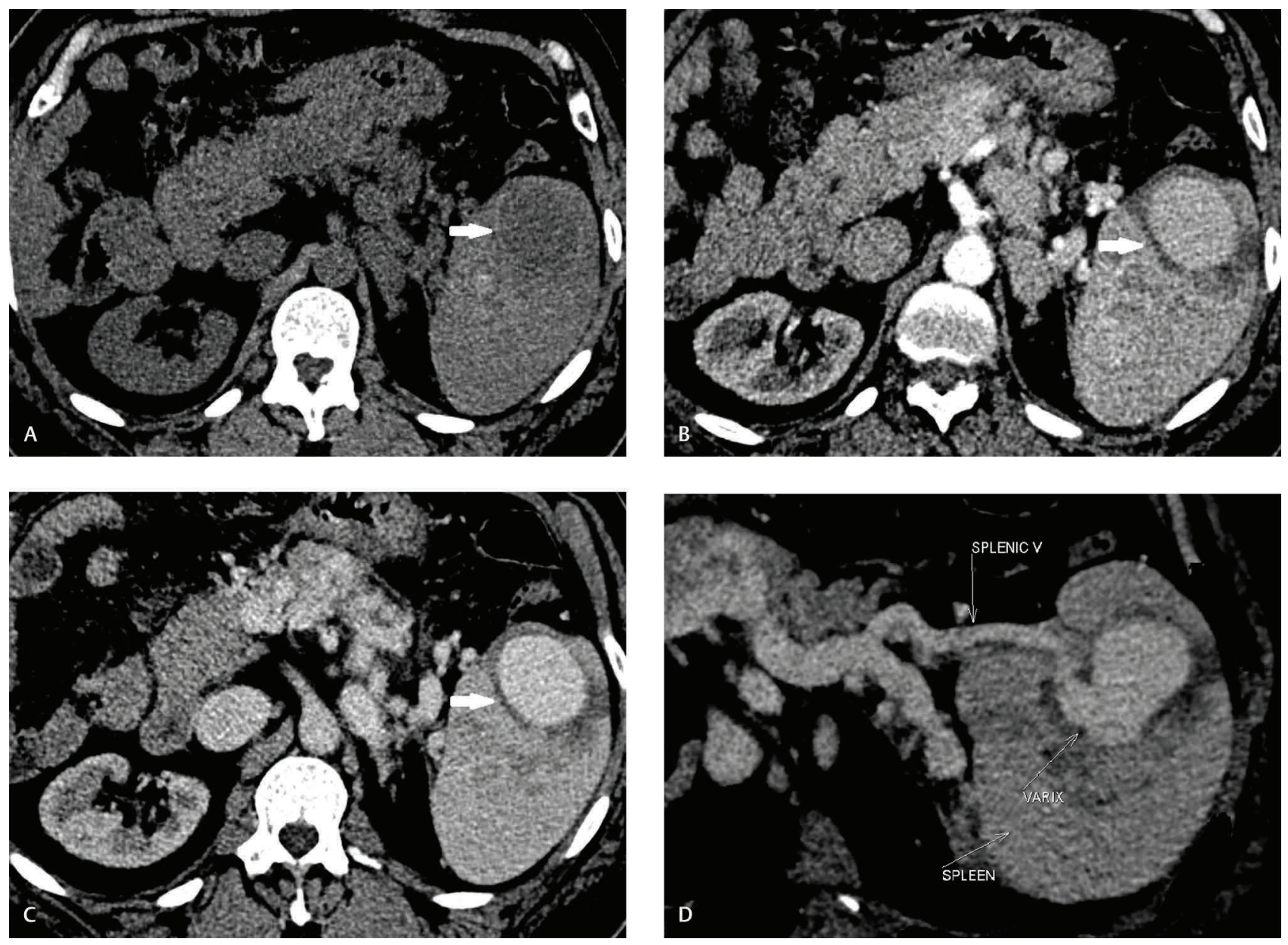

Fig. 3 Axial (A) nonenhanced computed tomography (NECT), (B) arterial, (C) venous, and (D) reformatted images of the spleen showing an intrasplenic varix (white arrow) paralleling the density of the splenic vein.

transvenous obliteration, or percutaneous transvenous embolization of the varices can be done. Familiarity with the afferent and efferent veins is of paramount importance, as the degree of difficulty in performing endovascular obliteration of gastric varices and the successful outcome of the procedures are directly related to the anatomic complexity of the varices. ${ }^{13}$ 

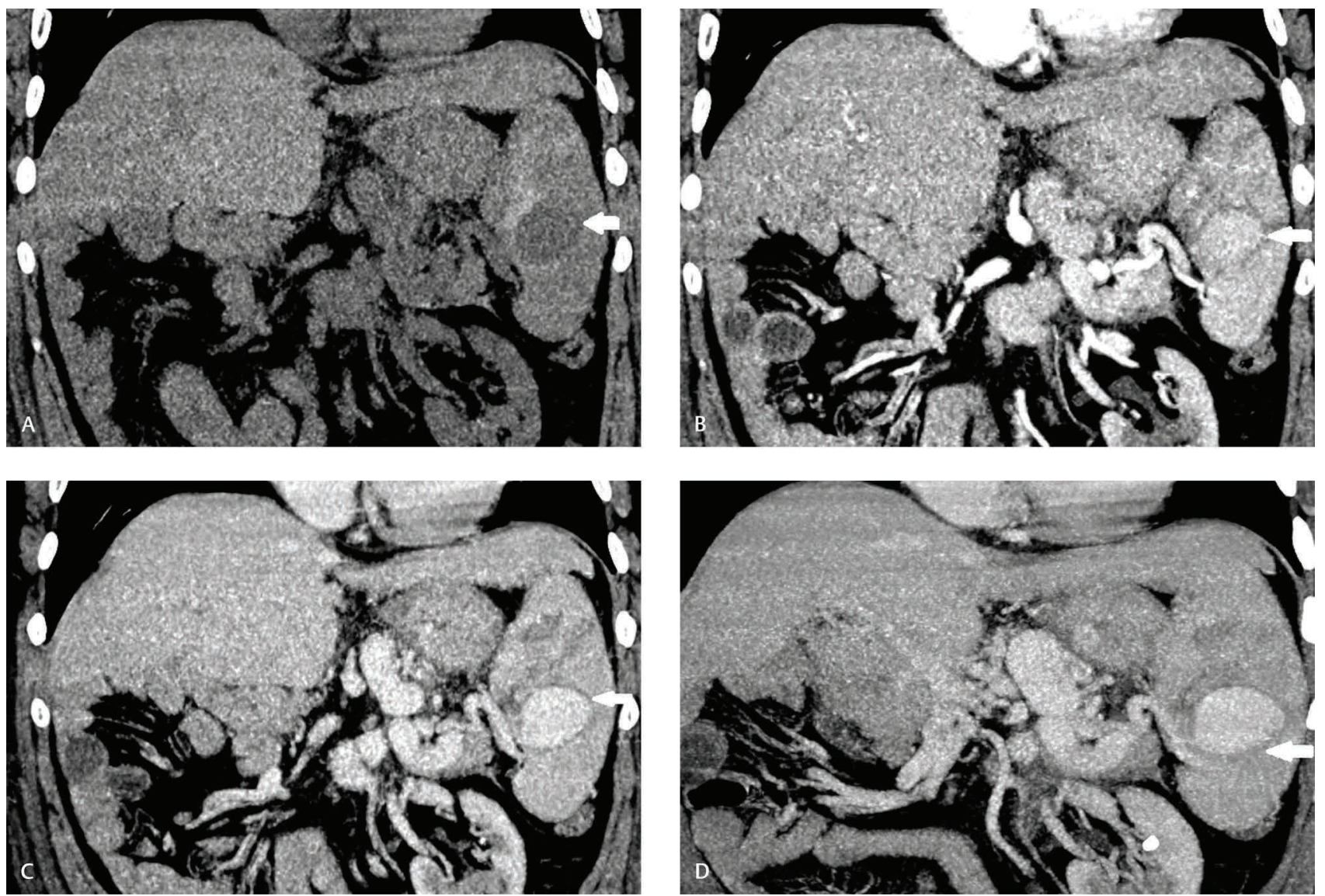

Fig. 4 Coronal computed tomography (CT) plain (A), arterial (B), venous (C) phase, and reformatted (D) images showing intrasplenic varix (white arrow) with contrast opacification paralleling that of the splenic vein.

Venous aneurysms of the splenic vein are rare. Congenital weakness of the venous wall, trauma, pancreatitis, and portal hypertension are possible etiologies. Few cases of extrasplenic splenic vein aneurysms have been reported. Gharabaghi et al reported a 12 -cm splenic vein aneurysm near the splenic hilum in a patient with chronic small bowel-type diarrhea. The huge splenic vein aneurysm and the backward flow into the superior mesenteric vein impeded the venous drainage of the small bowel. ${ }^{14}$ Ohhira et al reported a case of splenic vein aneurysm that progressively expanded in a patient with cirrhotic liver, portal hypertension, and hepatocellular carcinoma..$^{15}$ Tolgonay described a case of splenomegaly and splenic vein aneurysm due to a systemic infection in a patient with leukemia and no associated chronic liver disease. ${ }^{16}$ There are a few reports of splenic vein aneurysms in the puerperium; Parpaglioni et al reported a case of splenic vein aneurysm that had ruptured into the abdominal cavity producing retroperitoneal and intraperitoneal hemorrhage in the immediate postpartum period, which was managed surgically..$^{17}$

To the best of our knowledge, an intrasplenic varix has not been previously reported and is an extremely rare presentation of a well-known disease. This intrasplenic varix could be a direct complication of the increased portal venous pressure caused by chronic portal vein thrombosis, as in this case. But direct correlation with the severity of portal venous outflow obstruction is yet to be studied because of its rarity. In view of possible complications like aneurysmal rupture and thrombosis, surgery remains the treatment modality of choice for symptomatic patients with or without progressive expansion of the aneurysm diameter. In asymptomatic patients, as in our case, a regular radiological follow-up is recommended.

\section{Conflict of Interest}

None.

\section{References}

1 Bosch J, Groszmann RJ, Shah VH. Evolution in the understanding of the pathophysiological basis of portal hypertension: How changes in paradigm are leading to successful new treatments. J Hepatol 2015;62(1, Suppl):S121-S130

2 Viallet A, Huet PM, Marleau D, Villeneuve JP. Assessment of portal hemodynamics. Gastroenterology 1980;79(3):603-605

3 Bosch J, Abraldes JG, Berzigotti A, García-Pagan JC. The clinical use of HVPG measurements in chronic liver disease. Nat Rev Gastroenterol Hepatol 2009;6(10):573-582

4 Bosch J, Abraldes JG, Fernández M, García-Pagán JC. Hepatic endothelial dysfunction and abnormal angiogenesis: new targets in the treatment of portal hypertension. J Hepatol 2010;53(3):558-567

5 Schuppan D, Afdhal NH. Liver cirrhosis. Lancet 2008;371 (9615):838-851

6 Bosch J, Navasa M, Garcia-Pagán JC, DeLacy AM, Rodés J. Portal hypertension. Med Clin North Am 1989;73(4):931-953 
7 Harmanci O, Bayraktar Y. Clinical characteristics of idiopathic portal hypertension. World J Gastroenterol 2007;13 (13):1906-1911

8 Berzigotti A, Seijo S, Reverter E, Bosch J. Assessing portal hypertension in liver diseases. Expert Rev Gastroenterol Hepatol 2013;7(2):141-155

9 Thabut D, Shah V. Intrahepatic angiogenesis and sinusoidal remodeling in chronic liver disease: new targets for the treatment of portal hypertension? J Hepatol 2010;53(5):976-980

10 Menon KV, Kamath PS. Regional and systemic hemodynamic disturbances in cirrhosis. Clin Liver Dis 2001;5(3):617-627, viii

11 Cichoz-Lach H, Celiński K, Słomka M, Kasztelan-Szczerbińska B. Pathophysiology of portal hypertension. J Physiol Pharmacol 2008;59 (Suppl 2):231-238

12 Philips CA, Anand L, Kumar KN, Kasana V, Arora A. Rare, spontaneous trans-splenic shunt and intra-splenic collaterals with attendant splenic artery aneurysms in an adult patient with compensated cirrhosis and portal hypertension. Gastroenterol Rep (Oxf) 2015;3(2):162-166

13 Arora A, Rajesh S, Meenakshi YS, Sureka B, Bansal K, Sarin SK. Spectrum of hepatofugal collateral pathways in portal hypertension: an illustrated radiological review. Insights Imaging 2015;6(5):559-572

14 Gharabaghi MA, Yazdi NA, Abrishami Z, Amini S. Splenic vein aneurysm. BMJ Case Rep 2012;2012:bcr1020115007

15 Ohhira M, Ono M, Ohhira M, Matsumoto A, Ohta H, Namiki M. Case report: splenic vein aneurysm--report of a lesion that progressively expanded. Br J Radiol 1994;67(799):656-658

16 Tolgonay G, Ozbek SS, Oniz H, Süzer E, Yurdakul LO. Regression of splenic vein aneurysm following resolution of splenomegaly. J Clin Ultrasound 1998;26(2):98-102

17 Parpaglioni R, Metta E, Zagari A, Celleno D. Spontaneous splenic vein aneurysm rupture in the puerperium. Int J Obstet Anesth 2009;18(1):48-51 